\title{
Tongue strength and swallowing dynamics in chronic obstructive pulmonary disease
}

\author{
Isabella Epiu $\mathbb{1}^{1,2,3}$, Simon C. Gandevia ${ }^{1,2,3}$, Claire L. Boswell-Ruys ${ }^{1,2,3}$, Emma Wallace ${ }^{1,4}$, \\ Jane E. Butler ${ }^{1,2}$ and Anna L. Hudson ${ }^{1,2}$
}

${ }^{1}$ Neuroscience Research Australia, Sydney, NSW, Australia. ${ }^{2}$ University of New South Wales Sydney, NSW, Australia. ${ }^{3}$ Prince of Wales Hospital, Sydney, NSW, Australia. ${ }^{4}$ Flinders University, Adelaide, SA, Australia.

Corresponding author: Anna Hudson (a.hudson@neura.edu.au)

Shareable abstract (@ERSpublications)

In this novel study of swallowing in COPD, there was no difference in tongue strength when compared to healthy controls, and in COPD participants with airway invasion, the inhibitory reflex to airway occlusion in inspiratory muscles was delayed https://bit.ly/3h4EeKw

Cite this article as: Epiu I, Gandevia SC, Boswell-Ruys CL, et al. Tongue strength and swallowing dynamics in chronic obstructive pulmonary disease. ERJ Open Res 2021; 7: 00192-2021 [DOI: 10.1183/ 23120541.00192-2021].

\section{Abstract}

Copyright @The authors 2021

This version is distributed under the terms of the Creative Commons Attribution NonCommercial Licence 4.0. For commercial reproduction rights and permissions contact permissions@ersnet.org

Received: 16 March 2021 Accepted: 27 April 2021

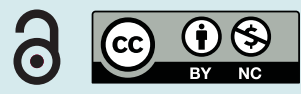

Background Swallowing disorders occur in COPD, but little is known about tongue strength and mastication. This is the first assessment in COPD of tongue strength and a test of mastication and swallowing solids (TOMASS).

Methods Anterior tongue strength measures were obtained in 18 people with COPD, aged $73 \pm 11$ years (mean $\pm \mathrm{sD}$ ), and 19 healthy age-matched controls, aged $72 \pm 6$ years. Swallowing dynamics were assessed using an eating assessment tool (EAT-10), timed water swallow test (TWST), and TOMASS. Swallowing measures were compared to an inhibitory reflex (IR) in the inspiratory muscles to airway occlusion (recorded previously in the same participants).

Results Tongue strength was similar between COPD and controls $(\mathrm{p}=0.715)$. Self-assessed scores of dysphagia EAT-10 were higher $(\mathrm{p}=0.024)$ and swallowing times were prolonged for liquids $(\mathrm{p}=0.022)$ and solids $(\mathrm{p}=0.003$ ) in the COPD group. During TWST, $30 \%$ of COPD group showed clinical signs of airway invasion (cough and wet voice), but none in the control group. For solids, the COPD group had $\sim 40 \%$ greater number of chews ( $\mathrm{p}=0.004)$, and twofold-higher number of swallows ( $\mathrm{p}=0.0496)$. Respiratory rate was $50 \%$ higher in COPD group than controls $(\mathrm{p}<0.001)$. The presence of an IR was not related to better swallowing outcomes, but signs of airway invasion were associated with a delayed IR.

Conclusion Dysphagia in stable COPD is not due to impaired anterior tongue strength, but rather swallowing-breathing discoordination. To address dysphagia, aspiration and acute exacerbations in COPD, therapeutic targets to improve swallowing dynamics could be investigated further.

\section{Introduction}

COPD, a major public health problem globally [1], is a progressive lung condition that causes airflow obstruction, chronic cough, shortness of breath and excess secretions in the airway.

Impaired swallowing (dysphagia), typically related to disruptions in breathing-swallowing coordination [2], has been documented in $20 \%$ of people with stable COPD [3], and in up to 56\% of COPD exacerbations [4]. Most healthy adults swallow during expiration, and the positive intra-thoracic pressure serves as an airway protective mechanism to expel any misdirected material from the laryngeal vestibule [5, 6]. In contrast, tachypnoeic COPD patients usually inspire during or immediately after swallowing, increasing their risk of aspiration and acute exacerbations [7].

COPD commonly occurs in advanced age $[4,8]$, but oropharyngeal muscle weakness as a mechanism for dysphagia has not been well explored. Fifteen percent of older adults (>65 years) have dysphagia [9] that is frequently attributed to oropharyngeal sarcopenia (loss of skeletal muscle mass and strength) [5]. 
As tongue strengthening, a treatment for oropharyngeal weakness, may prevent aspiration, the primary aim of this study was to evaluate tongue strength in COPD. Swallowing efficiency, mastication and clinical signs of airway invasion were also evaluated. The association between swallowing dynamics, participant anthropometrics and lung function were assessed.

We also assessed the relationship between swallowing and the short-latency inhibitory reflex (IR) in inspiratory muscles, which is a protective response evoked by airway occlusion [10]. We hypothesised COPD participants with alterations in IR may have clinical signs of aspiration and more severe dysphagia.

Methods

Ethics

University of New South Wales Human Research Ethics Committee approved the study (no. HC17762). Written informed consent was obtained. All procedures were conducted according to the Declaration of Helsinki (2013), except for database registration (clause 35).

\section{Participants}

Eligible participants had moderate to severe COPD or were healthy age-matched controls without chronic respiratory or neurological disease. The participants were either referred from colleagues at the Prince of Wales Private Hospital based on previous diagnosis of COPD or had previously volunteered in our laboratory and given permission to be contacted again. We used the GOLD criteria [11] to select people with moderate to severe COPD. Controls were recruited from the Neuroscience Research Australia Research Volunteers Registry or had previously volunteered. All participants with COPD were well at the time they were studied, i.e. during stable COPD.

In the same participants, we had previously measured the reflex responses to brief airway occlusion (see below for details) [12]. We compare the reflex results to the outcomes of the swallowing tests in the current study.

\section{Spirometry}

Pre-bronchodilator spirometry was performed while seated, using a hand-held spirometer (One Flow FVC Memo; Clement Clarke, Harlow, UK). At least three attempts of spirometry were performed, until two values of forced expiratory volume in $1 \mathrm{~s}\left(\mathrm{FEV}_{1}\right)$, force vital capacity (FVC) and peak expiratory flow (PEF) were within 10\% [13]. The highest values were presented as a percent predicted; derived using the European Respiratory Society Global Lung Initiative Calculator [14].

\section{Swallowing dynamics}

A standardised eating assessment tool (EAT-10) was used for self-assessment of swallowing [15]. High EAT-10 scores ( $>22$, from maximal score of 40 ) indicate profound dysphagia, EAT-10 scores $\geqslant 3$ suggest a possible issue with swallowing, and EAT-10 scores $\geqslant 15$ suggest a possible aspiration risk $[15,16]$.

Anterior tongue strength measures were obtained using the Iowa Oral Performance Instrument (IOPI), with participants instructed to elevate their tongue, and press the air-filled bulb on the hard palate following a standardised procedure [17]. The best of three consistent trials was recorded (two within 10\%).

Swallowing efficiency tests were performed using timed water swallow test (TWST) for liquids [18], and test of mastication and swallowing solids (TOMASS) for solids [19], with audio-visual recordings of the jaw and neck region. We monitored for cough, during or after (for $1 \mathrm{~min}$ ) the tests, and also signs of a wet-sounding gurgly voice. TWST was performed twice with $150 \mathrm{~mL}$ of water ( $>1$ min between tests). TOMASS was performed once, with participants instructed to eat $1 / 4$ of Arnott's Salada cracker $(5 \times 5 \mathrm{~cm})$ “as quickly and comfortably as possible”.

\section{Inspiratory muscle reflexes}

Comfortably seated participants wearing a nose clip breathed through a mouthpiece connected to a bacterial filter, pneumotachograph (Series 3813; Hans Rudolph, Kansas City, KS, USA) and a two-way valve (Series 2600; Hans Rudolph). The respiratory rate was calculated during quiet breathing. Then, a balloon valve in the inspiratory port of the two-way valve occluded the airway for $250 \mathrm{~ms}$ on random breaths until 30-40 occlusions were recorded [10, 12]. Surface electromyography activity (EMG) was recorded from the scalene muscles bilaterally using a standardised electrode placement [20], and over the right costal diaphragm at the seventh/eighth intercostal space with clear-trace ECG electrodes (ConMed Corp., Utica, NY, USA) [12] and the EMG signals were rectified and averaged across occlusions. 


\section{Statistical analysis}

Tongue strength and swallowing dynamics were compared between the COPD and control groups. Tongue strength results were compared with published normative data [17]. T-tests or Mann-Whitney rank-sum tests were used to compare swallowing results between and within the groups using Stata version 14 (StataCorp, College Station, TX, USA). Spearman and Pearson's correlations were used to analyse the associations between anthropometric and swallowing data. Normality (Shapiro-Wilk tests), and correlation tests were performed using GraphPad Prism 8.4.3. Data are expressed as mean \pm sD or median (interquartile range). Statistical significance was set at $\mathrm{p}<0.05$ in this exploratory study and Bonferroni corrections were not applied.

\section{Results}

We recruited 18 participants with moderate to severe COPD and 20 healthy controls (figure 1). One control participant was excluded due to inconsistent spirometry. Additionally, one COPD participant had an EAT-10 score of 16, and therefore did not proceed with TWST and TOMASS, but tongue strength was assessed. Two control participants did not have adequate reflex data for comparison with the swallowing tests (figure 1).

There were 11 female participants in each group (61\% (COPD) and 57.9\% (control)). Groups were matched for age $(73 \pm 11$ and $72 \pm 6$ years; table 1$)$. Spirometry parameters were significantly lower in the COPD group than in control, $\mathrm{FEV}_{1} / \mathrm{FVC}$ for COPD $50 \pm 16 \%$ and controls $79.9 \pm 5.7 \%$ ( $\mathrm{p}<0.001$ ). PEF was $164 \pm 55.1 \mathrm{~L} \cdot \mathrm{s}^{-1}$ and $289 \pm 85.2 \mathrm{~L} \cdot \mathrm{s}^{-1}$, respectively $(\mathrm{p}<0.001$; table 1$)$.

\section{Tongue strength assessment}

Anterior tongue strength was similar between the COPD and control groups (45.2 \pm 15.5 and $46.8 \pm 11.0 \mathrm{kPa}$, respectively, $\mathrm{p}=0.715$; table 1 ). Neither the COPD nor control group were underweight (body mass index $(\mathrm{BMI})<18.5 \mathrm{~kg} \cdot \mathrm{m}^{-2}$, table 1$)$. There were no associations of tongue strength with TWST time, $(\mathrm{p}=0.297$ and $\mathrm{p}=0.766)$, or TOMASS time, $(\mathrm{p}=0.746$ and $\mathrm{p}=0.120)$, for COPD and control groups, respectively (table 2); however, compared to weighted averages of previously published tongue strength data using the

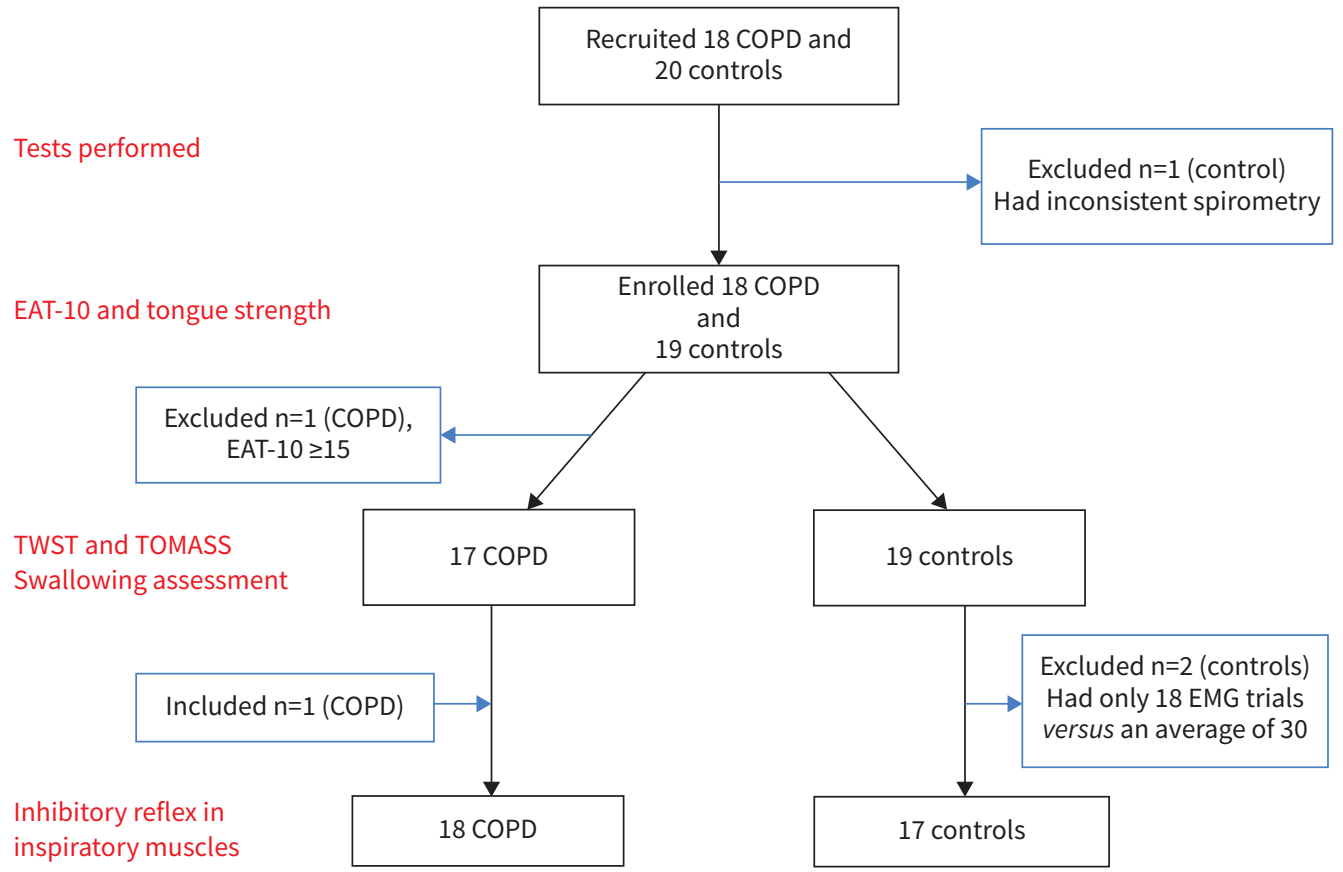

FIGURE 1 Enrolment of participants with COPD and age-matched controls for swallowing tests and comparison with previously published inhibitory reflex data [12]. An eating assessment tool (EAT-10) score (self-assessed score of dysphagia) of $\geqslant 15$ indicates a higher aspiration risk and we excluded such participants from the timed water swallow test (TWST) and test of mastication and swallowing of solids (TOMASS) assessments $[15,16]$. A score of $\geqslant 3$ indicates possible issue with swallowing, and here we proceeded with caution. EMG: electromyography. The timing of the tests performed is indicated on the left in red text. 
TABLE 1 Summary of anthropometric, respiratory and spirometry data

\begin{tabular}{|c|c|c|c|}
\hline & COPD $(n=18)$ & Control $(n=19)$ & $\mathrm{p}$-value \\
\hline Age years & $73 \pm 11$ & $72 \pm 6$ & 0.799 \\
\hline Female $\mathrm{n}(\%)$ & $11(61)$ & $11(58)$ & 0.842 \\
\hline $\mathrm{BMI} \mathrm{kg} \cdot \mathrm{m}^{-2}$ & $23.0(20.1-24.8)$ & $26.5(22.1-28.7)$ & 0.056 \\
\hline Smoking pack-years $\cdot$ day $^{-1}$ & $18.3(2.0-30.0)$ & $0.1(0.0-1.0)$ & $0.002^{*}$ \\
\hline $\mathrm{FEV}_{1} \mathrm{~L}$ & $1.2 \pm 0.4$ & $2.3 \pm 0.5$ & $<0.001^{\star}$ \\
\hline $\mathrm{FEV}_{1} \%$ pred & $55.7 \pm 18.4$ & $89.8 \pm 12.4$ & $<0.001^{\star}$ \\
\hline FVC L & $2.4 \pm 0.7$ & $2.9 \pm 0.7$ & 0.057 \\
\hline FVC \% pred & $80.4 \pm 20.4$ & $86.1 \pm 11.7$ & 0.310 \\
\hline PEF L.s ${ }^{-1}$ & $164 \pm 55.1$ & $289 \pm 85.2$ & $<0.001^{*}$ \\
\hline $\mathrm{FEV}_{1} / \mathrm{FVC} \%$ & $50.4 \pm 15.5$ & $79.9 \pm 5.7$ & $<0.001^{\star}$ \\
\hline Respiratory rate breaths $\cdot \mathrm{min}^{-1}$ & $18 \pm 4.8$ & $12 \pm 10.3$ & $<0.001^{\star}$ \\
\hline \multicolumn{4}{|c|}{$\begin{array}{l}\text { Data are presented as mean } \pm \mathrm{SD} \text { or median (interquartile range), unless otherwise stated. Anthropometric and } \\
\text { spirometry data as well as the eating assessment tool score, tongue strength and respiratory rate for COPD and } \\
\text { the age-matched control groups. t-tests and Mann-Whitney tests were used to compare between groups. } \\
\text { BMI: body mass index; FEV }{ }_{1} \text { : forced expiratory volume in } 1 \mathrm{~s} \text {; FVC: forced vital capacity; PEF: peak expiratory } \\
\text { flow. }{ }^{\star}: p<0.05 \text {. }\end{array}$} \\
\hline
\end{tabular}

TABLE 2 Correlations between eating assessment tool (EAT-10), tongue strength (IOPI), respiratory rate (RR) by spirometry, body mass index (BMI), timed water swallow test (TWST) and test of mastication and swallowing of solids (TOMASS) variables for the COPD and control groups

\begin{tabular}{|c|c|c|c|c|}
\hline & \multicolumn{2}{|l|}{ COPD } & \multicolumn{2}{|l|}{ Controls } \\
\hline & $\mathrm{R}(95 \% \mathrm{Cl})$ & p-value & $\mathrm{R}(95 \% \mathrm{Cl})$ & $\mathrm{p}$-value \\
\hline \multicolumn{5}{|l|}{ EAT-10 } \\
\hline $\mathrm{BMI} \mathrm{kg} \cdot \mathrm{m}^{-2}$ & $-0.014(-0.489-0.468)$ & 0.957 & $-0.180(-0.596-0.312)$ & 0.462 \\
\hline \multicolumn{5}{|l|}{ TWST } \\
\hline Total time s & $0.156(-0.364-0.602)$ & 0.546 & $0.115(-0.371-0.551)$ & 0.639 \\
\hline Number of swallows & $0.014(-0.470-0.491)$ & 0.957 & $0.367(-0.105-0.704)$ & 0.123 \\
\hline \multicolumn{5}{|l|}{ TOMASS } \\
\hline Number of swallows & $0.557(0.088-0.823)$ & $0.022^{*}$ & $-0.046(-0.501-0.430)$ & 0.852 \\
\hline Total time s & $0.218(-0.308-0.641)$ & 0.399 & $-0.043(-0.500-0.431)$ & 0.861 \\
\hline $\mathrm{FEV}_{1} / \mathrm{FVC}$ ratio $\%$ & $0.461(-0.023-0.770)$ & 0.054 & $-0.034(-0.492-0.438)$ & 0.889 \\
\hline $\mathrm{FEV}_{1} \mathrm{~L}$ & $0.057(-0.433-0.521)$ & 0.823 & $0.119(-0.367-0.554)$ & 0.627 \\
\hline $\mathrm{FEV}_{1} \%$ pred & $-0.147(-0.584-0.357)$ & 0.561 & $0.366(-0.120-0.711)$ & 0.123 \\
\hline \multicolumn{5}{|l|}{ IOPI } \\
\hline $\mathrm{BMI} \mathrm{kg} \cdot \mathrm{m}^{-2}$ & $-0.436(-0.773-0.092)$ & 0.092 & $0.233(-0.276-0.640)$ & 0.352 \\
\hline \multicolumn{5}{|l|}{ TWST } \\
\hline Total time s & $0.268(-0.259-0.672)$ & 0.297 & $-0.073(-0.521-0.406)$ & 0.766 \\
\hline Number of swallows & $0.478(-0.019-0.786)$ & 0.054 & $0.203(-0.290-0.611)$ & 0.404 \\
\hline Volume per swallow & $-0.478(-0.786-0.019)$ & 0.054 & $-0.203(-0.611-0.290)$ & 0.404 \\
\hline \multicolumn{5}{|l|}{ TOMASS } \\
\hline Number of chews & $-0.002(-0.494-0.491)$ & 0.993 & $-0.323(-0.686-0.168)$ & 0.177 \\
\hline Number of swallows & $0.091(-0.420-0.558)$ & 0.727 & $0.327(-0.163-0.689)$ & 0.172 \\
\hline Total time s & $-0.085(-0.554-0.426)$ & 0.746 & $-0.369(-0.712-0.117)$ & 0.120 \\
\hline \multicolumn{5}{|c|}{ 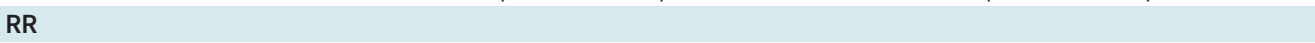 } \\
\hline EAT-10 & $0.517(0.051-0.798)$ & $0.028^{\star}$ & $0.126(-0.361-0.559)$ & 0.607 \\
\hline TWST total time s & $0.231(-0.295-0.650)$ & 0.369 & $0.069(-0.410-0.518)$ & 0.781 \\
\hline TOMASS total time s & $0.112(-0.403-0.573)$ & 0.667 & $-0.224(-0.625-0.270)$ & 0.357 \\
\hline TWST volume per second & $-0.212(-0.628-0.200)$ & 0.415 & $-0.107(-0.535-0.365)$ & 0.664 \\
\hline TWST volume per swallow & $-0.199(-0.620-0.311)$ & 0.443 & $-0.110(-0.537-0.363)$ & 0.655 \\
\hline $\mathrm{FEV}_{1} \mathrm{~L}$ & $-0.216(-0.620-0.279)$ & 0.389 & $-0.149(-0.565-0.327)$ & 0.542 \\
\hline FEV $_{1} \%$ pred & $0.176(-0.317-0.594)$ & 0.486 & $-0.052(-0.495-0.412)$ & 0.833 \\
\hline
\end{tabular}


a)

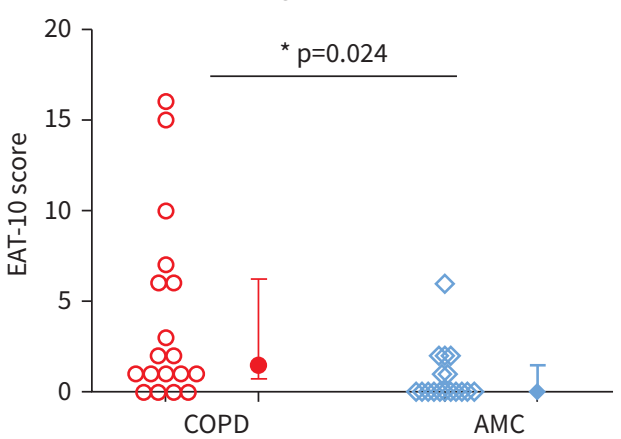

b)

EAT-10 score and respiratory rate

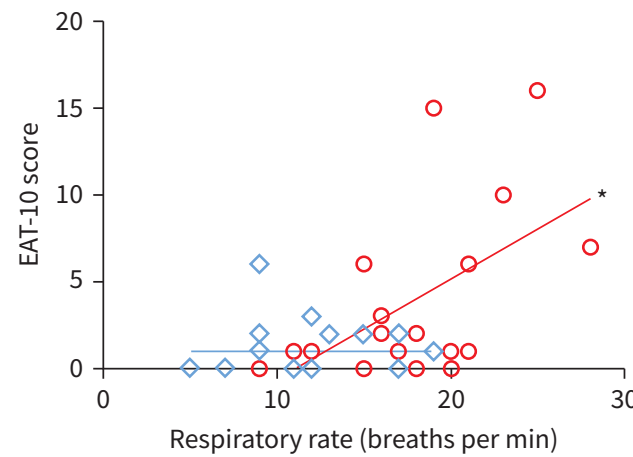

FIGURE 2 a) Individual data (open symbols) and median and interquartile range (closed symbols) for participants with COPD $(n=18)$ and age-matched control (AMCs) $(n=19)$. b) Correlations between respiratory rate and eating assessment tool (EAT-10) for those with COPD (red) and AMCs (blue). *: $p<0.05$ for t-test comparisons and Spearman's correlations within the COPD group.

IOPI [17], both COPD and control groups had lower tongue strengths compared to older controls aged $>60$ years, $57.4 \pm 13.0 \mathrm{kPa}$ [17] $(\mathrm{p}=0.005$ versus $\mathrm{p}=0.001$, respectively), and younger controls aged 20-39 years, $65.7 \pm 13.0 \mathrm{kPa}[17]$ ( $\mathrm{p}<0.001$ versus $\mathrm{p}<0.001$, respectively).

a)

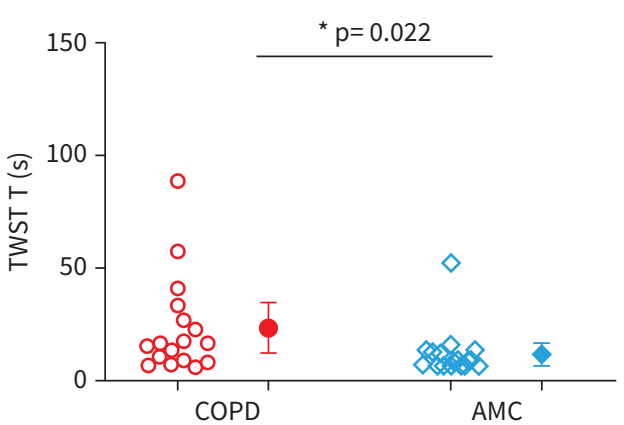

c)

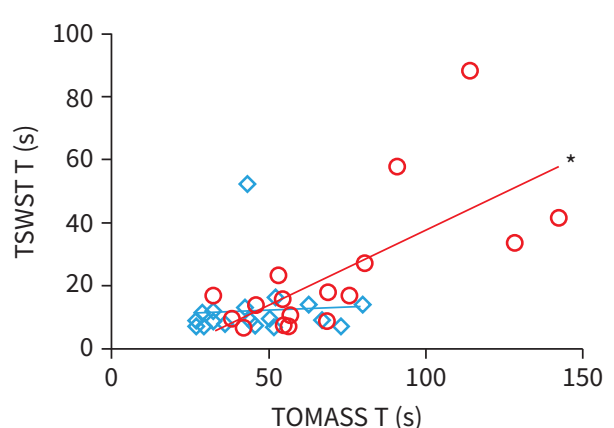

b)

TOMASS T

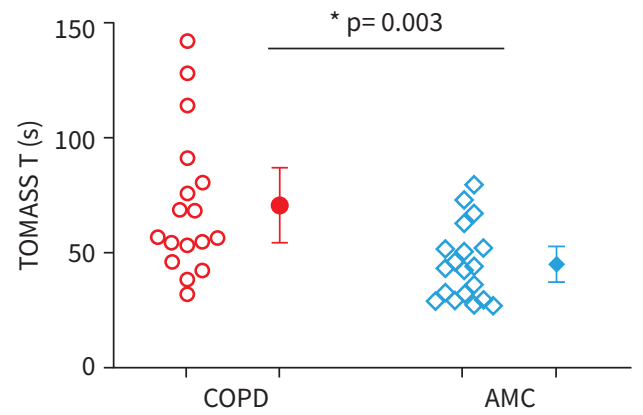

d) EAT-10 score versus TOMASS number of swallows

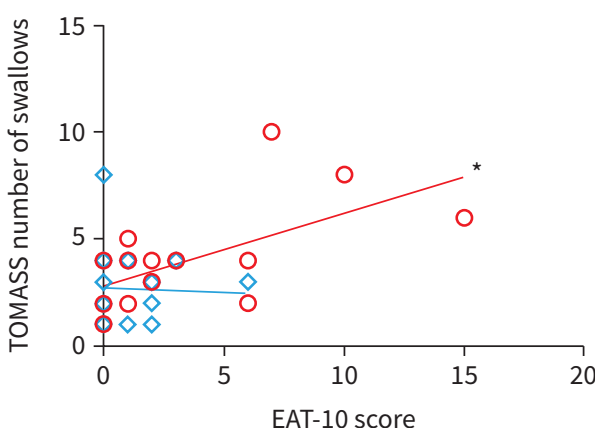

FIGURE 3 Individual data (open symbols) and mean (95\% Cl) (closed symbols) for participants with COPD $(n=17)$ and age-matched controls (AMCS) $(n=19)$ for a) timed water swallow test (TWST) and b) test for mastication and swallowing of solids (TOMASS) times (T). Correlations between C) TOMASS and TWST T and d) eating assessment tool (EAT-10) score and TOMASS number of swallows for those with COPD (red) and AMCs (blue). ${ }^{*}$ : $p<0.05$ for t-test comparisons and Spearman's correlations within the COPD group. 


\begin{tabular}{|c|c|c|c|}
\hline & COPD $(n=17)$ & Control $(n=19)$ & p-value \\
\hline \multicolumn{4}{|l|}{ TWST for liquids } \\
\hline Total time s & $17.0(9.4-27.3)$ & $9.0(7.5-13.2)$ & $0.022^{\star}$ \\
\hline Number of swallows & $7.0(7.0-10.0)$ & $6.0(5.0-8.0)$ & 0.053 \\
\hline Volume per second $\mathrm{mL} \cdot \mathrm{s}^{-1}$ & $10.7 \pm 6.6$ & $15.6 \pm 5.1$ & $0.019^{*}$ \\
\hline Volume per swallow mL & $21.4(15.0-21.4)$ & $25.0(18.8-30.0)$ & 0.053 \\
\hline Time per swallow s & $2.0(1.5-2.6)$ & $1.4(1.3-1.8)$ & $0.020^{*}$ \\
\hline \multicolumn{4}{|l|}{ TOMASS for solids } \\
\hline Total time s & $56.7(53.1-80.4)$ & $43.4(29.3-52.1)$ & $0.003^{*}$ \\
\hline Number of swallows & $4.0(2.0-4.0)$ & $2.0(2.0-4.0)$ & $0.0496^{*}$ \\
\hline Number of bites & $4.0(3.0-5.0)$ & $3.0(2.0-3.0)$ & 0.079 \\
\hline Number of chews & $68.5 \pm 22.5$ & $48.7 \pm 13.2$ & $0.004^{*}$ \\
\hline Chews per bite & $18.2(15.8-23.0)$ & $18.0(12.8-27.0)$ & 0.739 \\
\hline Swallows per bite & $1.0(0.8-1.3)$ & $1.0(0.7-1.3)$ & 0.547 \\
\hline Time per bite $s$ & $18.7(16.3-22.7)$ & $14.6(10.8-21.2)$ & 0.132 \\
\hline Time per chew s & $1.01(0.8-1.2)$ & $0.9(0.8-1.0)$ & 0.199 \\
\hline Time per swallow s & $18.7(13.6-21.0)$ & $14.6(13.0-26.9)$ & 0.635 \\
\hline
\end{tabular}

\section{Swallowing efficiency and mastication assessment}

EAT-10 scores were significantly higher in the COPD group $(1.5(1.0,6.0))$ than in controls $(0(0,2.0))$, ( $p=0.024$; figure 2), indicative of a higher level of self-reported dysphagia in COPD. Among the COPD participants, 7 ( $40 \%)$, compared to $2(\sim 11 \%)$ control participants had EAT-10 scores $\geqslant 3$, suggesting the presence of dysphagia. Total TWST times were 50\% longer, and TOMASS times $31 \%$ longer in the COPD group than in the control group, $(\mathrm{p}=0.022$ and $\mathrm{p}=0.003$; figure $3 \mathrm{a}$ and $\mathrm{b}$ and table 3$)$. Total TWST times correlated positively with TOMASS times in the COPD group ( $R=0.686, p=0.003$; figure $3 c)$, but not the control group $(\mathrm{R}=0.318, \mathrm{p}=0.185)$. The EAT-10 score correlated positively with higher TOMASS number of swallows in the COPD group $(R=0.557, p=0.022$, figure $3 d)$, but not the control group $(R=$ $-0.046, \mathrm{p}=0.852$ ). No association was observed between EAT-10 and BMI, TWST or spirometry in the COPD or control groups (table 2).

The COPD group took a longer time to swallow $150 \mathrm{~mL}$ of water, taking $17.0 \mathrm{~s}(9.4,27.3)$ compared to $9.0 \mathrm{~s}(7.5,13.2)$ in the control group $(\mathrm{p}=0.022)$. COPD group had a lower liquid swallowing efficiency than control group $10.7 \pm 6.6 \mathrm{~mL} \cdot \mathrm{s}^{-1}$ versus $15.6 \pm 5.1 \mathrm{~mL} \cdot \mathrm{s}^{-1}(\mathrm{p}=0.019)$. The time per liquid swallow was $2.0 \mathrm{~s}(1.5,2.6)$ in the COPD group compared to $1.4 \mathrm{~s}(1.3,1.8)$ in the control group $(\mathrm{p}=0.020$; table 3$)$. There was a negative correlation between the TWST time and $\mathrm{FEV}_{1}$ \%predicted in the COPD group $(\mathrm{R}=$ -0.721 , $\mathrm{p}=0.002$; figure $4 \mathrm{c})$ but not in the control group $(\mathrm{R}=-0.179, \mathrm{p}=0.464)$. This indicates a lower efficiency of swallowing liquids in more severe COPD.

TOMASS times were longer in the COPD group $(56.7 \mathrm{~s}(53.1,80.4))$ than the controls $(43.4 \mathrm{~s}(29.3,52.1)$, $\mathrm{p}=0.003$; table 3$)$. The COPD group had a higher number of chews $(68.5 \pm 22.5)$ than the control group (48.7 \pm 13.2$)$ ( $p=0.004$; table 3$)$. Total number of swallows was twofold higher in the COPD group than the control group ( $\mathrm{p}=0.0496$; table 3 ). There was no difference between the COPD and control groups in the other TOMASS variables (table 3). A negative correlation was observed between TOMASS time and $\mathrm{FEV}_{1}$ \%predicted in the COPD group $(\mathrm{R}=-0.498, \mathrm{p}=0.044$; figure $4 \mathrm{~d})$, but not the control group $(\mathrm{R}=$ $-0.219, p=0.367$; figure $4 d$ ).

\section{Events during the swallowing tests}

Five of the COPD participants (29.4\%) had clinical signs of airway invasion during the TWST (i.e. events: cough and wet voice), but none was observed in the control group. Among the COPD participants 1 of 17 (6\%) coughed during, 3 of 17 (18\%) coughed after the TWST, and 1 of 17 (6\%) had a wet voice quality immediately after the test, which recovered within a minute. No clinical signs of airway invasion were noted during or after the TOMASS. In the COPD group, 2 of 17 (12\%) had dentures, which they kept in during the tests, and another participant chewed the cracker with front teeth as they did not have molars. There were no differences in the total time for TOMASS $(p=0.169)$, TWST $(p=0.114)$, EAT-10 $(p=0.085)$, 

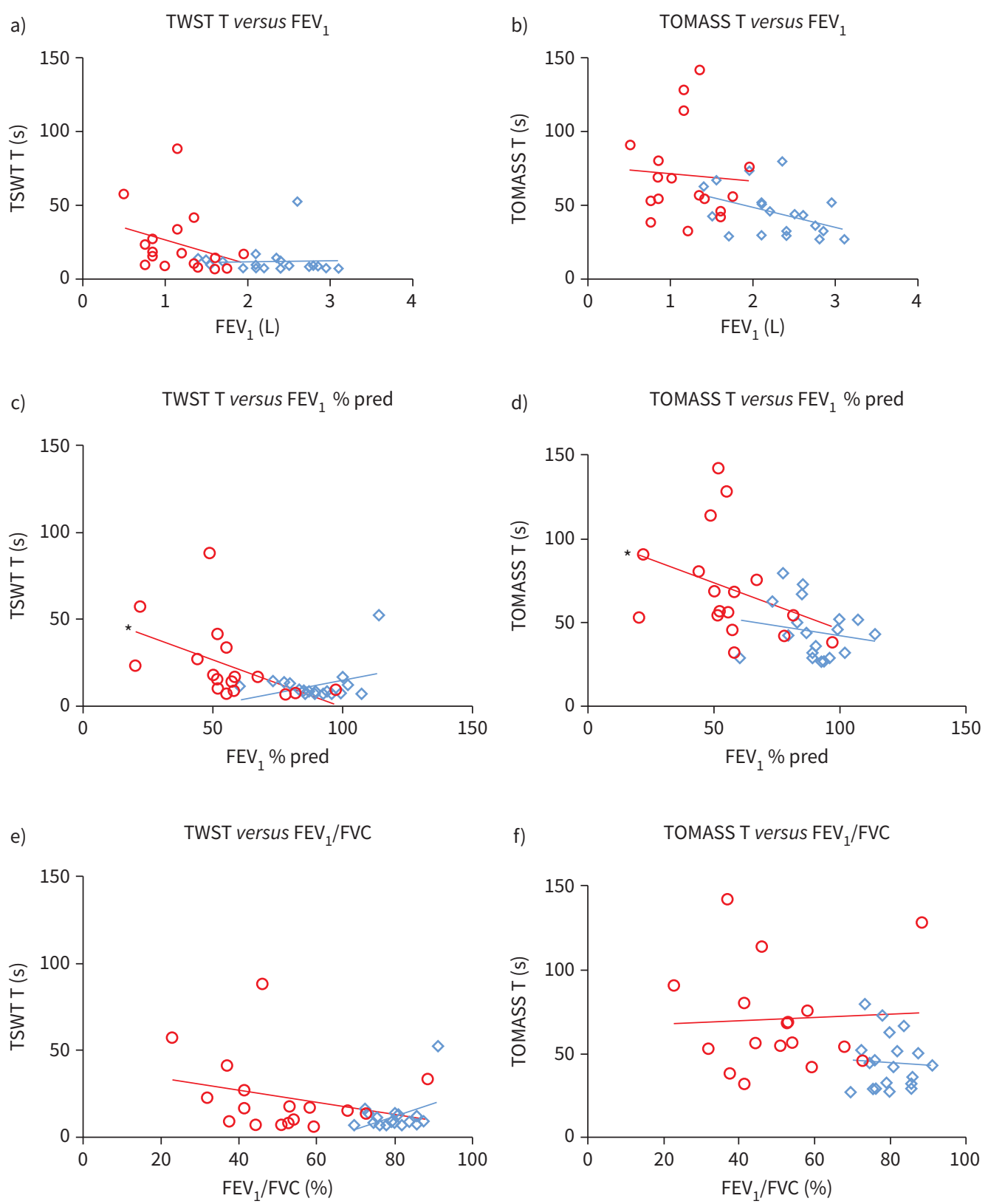

FIGURE 4 a-f) Correlations between total times (T) for test for mastication and swallowing of solids (TOMASS) and timed water swallow test (TWST) and the spirometry measures for those with COPD (red) and age-matched controls (AMCS) (blue). We used Spearman and Pearson correlation (R) to compute the nonparametric and parametric correlations, respectively. $\mathrm{FEV}_{1}$ : forced expiratory volume in $1 \mathrm{~s}$; FVC: forced vital capacity. *: $p<0.05$ for Spearman's correlations within the COPD group.

or tongue strength $(\mathrm{p}=0.805)$ tests between patients with or without events, except for greater number of bites ( $5 \pm 1.1$ versus $3 \pm 1.2, p=0.002$ ), and fewer chews per bite in the TOMASS test; $22.3(16.1,25.3$ ) versus $14.6(10.0,15.8)$, shown in table 4.

\section{Respiratory rate correlations}

The COPD group had a 50\% higher respiratory rate than the control group, with 18 versus 12 breaths per minute $(\mathrm{p}<0.001)$. The EAT-10 score positively correlated with respiratory rates in the COPD group $(\mathrm{R}=0.517, \mathrm{p}=0.028)$, but not the control group $(\mathrm{R}=0.126, \mathrm{p}=0.607)$. No association was observed between the respiratory rate and total time of TWST and TOMASS in either the COPD $(\mathrm{R}=0.231, \mathrm{p}=0.369$ and $\mathrm{R}=0.112$, $\mathrm{p}=0.667$, respectively) or age-matched control groups $(\mathrm{R}=0.069, \mathrm{p}=0.781$ and $\mathrm{R}=-0.224$, 


\section{TABLE 4 Within-group analysis of signs of airway invasion (events)}

\begin{tabular}{|c|c|c|c|}
\hline & No events & Yes events & p-value \\
\hline \multicolumn{4}{|l|}{ TWST for liquids } \\
\hline Sample size & 12 & 5 & \\
\hline Total time s & $13.8(8.1-20.6)$ & $27.3(15.7-41.6)$ & 0.114 \\
\hline Number of swallows & $7.7 \pm 2.46$ & $10.8 \pm 4.1$ & 0.067 \\
\hline Volume per second $\mathrm{mL} \cdot \mathrm{s}^{-1}$ & $12.5 \pm 6.8$ & $6.2 \pm 3.9$ & 0.073 \\
\hline Volume per swallow mL & $21.7 \pm 16.9$ & $15.6 \pm 8.5$ & 0.124 \\
\hline Time per swallow s & $1.7(1.4-2.4)$ & $2.2(2.0-2.6)$ & 0.092 \\
\hline \multicolumn{4}{|l|}{ TOMASS for solids } \\
\hline Sample size & 12 & 5 & \\
\hline Total time s & $63.8 \pm 26.1$ & $87.3 \pm 40.6$ & 0.169 \\
\hline Number of swallows & $7.0(5.5-10.0)$ & $10.0(7.0-14.0)$ & 0.134 \\
\hline Number of bites & $3.1 \pm 1.2$ & $5.4 \pm 1.1$ & $0.002^{\star}$ \\
\hline Number of chews & $65.7 \pm 18.7$ & $75.2 \pm 31.5$ & 0.445 \\
\hline Chews per bite & $22.3(16.1-25.3)$ & $14.6(10.0-15.8)$ & $0.011^{\star}$ \\
\hline Swallows per bite & $1.2 \pm 0.6$ & $0.9 \pm 0.9$ & 0.330 \\
\hline Time per bite s & $19.0(18.0-27.4)$ & $13.4(11.5-16.3)$ & 0.058 \\
\hline Time per chew s & $1.0 \pm 0.3$ & $1.2 \pm 0.4$ & 0.225 \\
\hline Time per swallow s & $18.4(15.4-24.7)$ & $14.2(13.6-20.1)$ & 0.598 \\
\hline \multicolumn{4}{|l|}{ IOPI } \\
\hline Sample size & 12 & 5 & \\
\hline Tongue strength kPa & $42.6 \pm 16.1$ & $50.2 \pm 15.6$ & 0.805 \\
\hline \multicolumn{4}{|l|}{ EAT-10 } \\
\hline Sample size & 12 & 5 & \\
\hline Self-assessed dysphagia score & $1.0(0.5-2.0)$ & $6.0(3.0-10.0)$ & 0.085 \\
\hline \multicolumn{4}{|l|}{ IR scalenes } \\
\hline Sample size & 9 & 4 & \\
\hline IR onset ms & $56.2 \pm 9.7$ & $60.9 \pm 11.2$ & 0.461 \\
\hline IR duration $\mathrm{ms}$ & $62.3 \pm 28.6$ & $75.2 \pm 32.6$ & 0.485 \\
\hline $\mathrm{IR}$ area $\mathrm{mV} \cdot \mathrm{ms}$ & $14.5(10.0-17.7)$ & $15.3(13.3-32.3)$ & 0.643 \\
\hline IR peak \% & $62.1(60.4-70.1)$ & $60.2(47.3-66.7)$ & 0.643 \\
\hline IR peak time $\mathrm{ms}$ & $82.0(76.0-94.5)$ & $93.5(89.3-108)$ & 0.142 \\
\hline \multicolumn{4}{|l|}{ IR diaphragm } \\
\hline Sample size & 6 & 3 & \\
\hline IR onset ms & $58.0 \pm 7.9$ & $86.0 \pm 14.1$ & $0.007^{\star}$ \\
\hline IR duration ms & $71.2 \pm 24.5$ & $63.7 \pm 34.9$ & 0.715 \\
\hline $\mathrm{IR}$ area $\mathrm{mV} \cdot \mathrm{ms}$ & $19.3 \pm 8.9$ & $21 \pm 18.2$ & 0.854 \\
\hline IR peak \% & $62.4(53.6-64.7)$ & $61.4(36.8-78.8)$ & 0.796 \\
\hline IR peak time $\mathrm{ms}$ & $101(95.0-110)$ & $104(104-115)$ & 0.294 \\
\hline \multicolumn{4}{|c|}{$\begin{array}{l}\text { Data are presented as } \mathrm{n} \text {, median (interquartile range) or mean } \pm \mathrm{SD} \text {, unless otherwise stated. Within-group } \\
\text { analysis of participants with COPD who had clinical signs of airway invasion (events) during timed water } \\
\text { swallow test (TWST) test for swallowing liquids. The presence of an inhibitory reflex (IR) in the scalene and } \\
\text { diaphragm muscles was assessed using } 2 \text { sD criteria, i.e. airway occlusion evoked a decrease in inspiratory } \\
\text { muscle electromyographic activity (EMG) of } 2 \mathrm{sD} \text { below pre-occlusion EMG levels, which lasted } \geqslant 10 \text { ms [12]. } \\
\text { TOMASS: test of mastication and swallowing solids; IOPI: lowa Oral Performance Instrument; EAT-10: eating } \\
\text { assessment tool. *: } \mathrm{p}<0.05 \text { for t-test comparisons and Spearman's correlations within the COPD group. }\end{array}$} \\
\hline
\end{tabular}

$\mathrm{p}=0.357$, respectively; table 2). No correlations were observed between respiratory rate and TWST volume per swallow or volume per second, and there was no association with $\mathrm{FEV}_{1}$ \%predicted or $\mathrm{FEV}_{1}$, in either the COPD or control groups (table 2).

Within-group comparison of swallowing data with inspiratory muscle reflexes

IRs were observed in the COPD and control groups, but not in all participants and muscles [12]. The short-latency IR in either the scalene or diaphragm muscles was present in 15 of 18 (83\%) participants with COPD versus 7 of 17 (41\%) participants in the control group ( $p=0.010)$ [12]. Participants in the COPD group who had events during TWST had a delayed IR onset time in the diaphragm, when compared to those who did not have signs of airway invasion, (85 $\pm 14.1 \mathrm{~ms}$ versus $58 \pm 7.9 \mathrm{~ms}, \mathrm{p}=0.007)$. No other parameter of the reflex differed between participants with or without clinical signs of airway invasion (table 4). 
Discussion

We have shown no difference in tongue strength between stable COPD and healthy age-matched controls, despite an increased level of dysphagia in the COPD group. This suggests that anterior tongue strength is not the mechanism of dysphagia in this COPD group. Prolonged times for swallowing both solids and liquids in the COPD group compared to control indicate that the COPD group had a reduced swallowing efficiency and impaired masticatory ability. Longer times in both swallowing tests were associated with COPD severity. Additionally, a greater peak of the reflex inhibition in inspiratory muscles positively correlated with COPD severity [12].

Disruption to breathing-swallowing coordination is the most common cause of dysphagia in COPD [2123] and would be aggravated by a higher respiratory rate seen here and by others [8, 24]. The COPD participants had a 50\% higher respiratory rate, greater number of swallows (solids and liquids), and $~ 30 \%$ showed clinical signs of airway invasion (cough and wet voice), but none in the control group.

In contrast to our hypothesis, the presence of an inhibitory reflex was not related to better swallowing function, but rather we did observe a delayed onset of the IR in participants who showed clinical signs of airway invasion.

Dysphagia in COPD

Swallowing problems are considered a major risk factor for acute exacerbations of COPD (AECOPD) which present as an abrupt worsening of COPD symptoms, compromised lung function, decreased ventilation-perfusion ratios, and lower oxygen saturation [25, 26]. Dysphagia observed here with the bedside TWST and TOMASS is consistent with video-fluoroscopy studies in COPD, which reveal that airway penetration or aspiration is associated with tachypnoea, reduced hyoid elevation, post-swallow pharyngeal residue and more frequent hospitalisations [27, 28]. Aspiration may contribute to a higher morbidity in people with COPD by aggravating AECOPD, which together reduce the quality of life, and increase health-related costs [11, 28-30].

Poor swallowing efficiency is linked to a higher risk of post-swallow aspiration, especially in the presence of shortness of breath. Changes in ventilatory pattern can alter swallowing and compromise swallowing coordination [5, 31-33]. Tachypnoea may lead to a shorter pause (typical apnoea period of 0.5-1 s), and higher chance of a post-swallow inspiration which increases the risk of aspiration [27], as may have occurred in $\sim 30 \%$ of participants in the COPD group who had a cough or wet voice. However, a limitation of this study is that silent aspirations would have been missed during the TWST and TOMASS tests. To detect silent aspirations specialised evaluations are required (i.e. video-fluoroscopy and/or endoscopy).

The higher number of swallows for solids in the COPD group may indicate the presence of pharyngeal residue, prompting additional swallows. This aligns with previous research that showed pharyngeal residue in COPD patients with instrumental assessment [28]. Additionally, the COPD group swallowed less liquid (volume $\cdot \mathrm{s}^{-1}$ ) than controls, which may be a compensatory mechanism to enhance swallowing safety. The prolonged overall time for swallowing solids in the COPD group is probably due to the increased number of chews or reduced swallowing efficiency. This may be linked to a greater self-awareness of their difficulty swallowing as indicated by the high EAT-10 score in the COPD group. While an EAT-10 $\geqslant 3$ indicates swallowing difficulties in our elderly participants, another potential limitation is that the EAT-10 score may have been affected by recall bias in which participants reported less severe swallowing difficulties. Nonetheless, the overall EAT-10 scores were higher in COPD than control groups.

A second possibility for the prolonged time for swallowing solids in the COPD group may be oropharyngeal weakness, reduced masticatory efficiency, or poor dentition with $12 \%$ of the COPD group reporting poor dentition. We did not measure bite strength, but anterior tongue strength was comparable in both groups, as was the strength of the inspiratory muscles (maximal inspiratory pressure) which had been measured previously in the same participants [12].

Swallowing dynamics and the inhibitory reflex

The inspiratory muscle IR in response to airway occlusion was more prevalent in COPD compared to control group [12]. Here, the same COPD group also had higher EAT-10 scores, and lower swallowing efficiency of liquids and solids. Previous findings from repetitive saliva swallowing tests also suggest that abnormal swallowing reflexes were increased in COPD and those predisposed to exacerbations [8].

In COPD participants who showed clinical signs of airway invasion when swallowing water, the onset time for the IR in the diaphragm was longer, compared to those with COPD who did not exhibit signs of 
airway invasion. If the onset of the IR is delayed, then the reflex may be less protective against aspiration. In other words, a delayed decrease in negative (inspiratory) thoracic pressure may allow pharyngeal residue to be sucked into the airway.

While the sample sizes for these analyses were small, a delayed IR may be a marker to identify those at risk of AECOPD, if delayed inspiratory muscle reflexes can aggravate underlying swallowing problems indicated clinically by a cough, wet voice and/or aspiration. This needs to be explored further. As the IR is believed to be mediated by intramuscular receptors in the inspiratory muscles rather than airway mucosa or lung afferents [10, 34], any delay in the onset of IR is unlikely to be explained by any decrease in pharyngeal sensation [35]. In future studies, the relationship between the IR as a possible airway protective mechanism and the occurrence of airway invasion should be evaluated using endoscopy or video-fluoroscopy to provide clearer insight into this relationship.

\section{Tongue strength and dysphagia}

Dysphagia can be due to impaired neurological, muscular or psychogenic components of deglutination, and most dysphagia symptoms in COPD are related to impaired pharyngeal protective mechanisms [36]. Oropharyngeal dysphagia affects more than $60 \%$ of elderly institutionalised patients [5], commonly associated with age-related atrophy of the tongue, geniohyoid muscle, and the pharynx [37-39]. We found no difference in the tongue strength between the COPD and healthy controls (mean ages 73 and 72 years for the COPD and control groups, respectively), both lower than weighted averages of older ( $>60$ years) and young (20-39 years) controls [17]. While we enrolled people with stable COPD, some reports have shown that oropharyngeal dysphagia is more common during AECOPD [25]. Additionally, the similar $\mathrm{BMI}$ in the COPD and control groups suggests our COPD group did not have cachexia. Therefore, to address these complex swallowing dynamics in stable COPD, improving respiratory-swallowing coordination (e.g. using biofeedback, as piloted in a head and neck cancer cohort [40]) rather than tongue strengthening exercises may be more beneficial.

\section{Conclusion}

Our results confirm that people with COPD have swallowing difficulties, but decreased anterior tongue strength is unlikely to be a contributing factor in stable COPD. The higher incidence of airway invasion in COPD, a factor that may lead to frequent AECOPD, was linked to a delayed IR in the inspiratory muscles. Training methods to improve swallowing-breathing coordination, and the impaired occlusion reflex could be implemented to reduce exacerbations, hospitalisation costs and improve the quality of life for people with COPD.

Acknowledgements: We thank Ranasinghe H.C. Lewis at the Prince of Wales Hospital, who reviewed the spirometry data. We also thank Chanelle Basha and Sean N.J. Archer, who collected some swallowing and reflex data, and Karen Peebles from Macquarie University, for supporting C.B. and S.A.

Author contributions: All authors contributed to the study conception. I. Epiu, A.L. Hudson and C. Boswell-Ruys performed the experiment at Neuroscience Research Australia. I. Epiu analysed the data and drafted the manuscript. All authors interpreted the data and revised the manuscript. All authors approved the final version of the manuscript.

Conflict of interest: I. Epiu has nothing to disclose. S.C. Gandevia reports receiving a fellowship from the NHMRC. C.L. Boswell-Ruys has nothing to disclose. E. Wallace has nothing to disclose. J.E. Butler has nothing to disclose. A.L. Hudson reports grants from NHMRC and the Rebecca L. Cooper Foundation during the conduct of the study.

Support statement: This work was supported by the National Health and Medical Research Council (NHMRC), Australia (1138920) and the Rebecca L. Cooper Medical Research Foundation. I. Epiu is funded by a UNSW Scientia PhD Scholarship; S.C. Gandevia and J.E. Butler are supported by NHMRC Fellowships. Funding information for this article has been deposited with the Crossref Funder Registry.

\section{References}

1 Vestbo J, Hurd SS, Agusti AG, et al. Global strategy for the diagnosis, management, and prevention of chronic obstructive pulmonary disease: GOLD executive summary. Am J Respir Crit Care Med 2013; 187: 347-365.

2 Cvejic L, Bardin PG. Swallow and aspiration in chronic obstructive pulmonary disease. Am J Respir Crit Care Med 2018; 198: 1122-1129.

3 Robinson DJ, Jerrard-Dunne P, Greene Z, et al. Oropharyngeal dysphagia in exacerbations of chronic obstructive pulmonary disease. Euro Geria Med 2011; 2: 201-203. 
4 Mokhlesi B, Logemann JA, Rademaker AW, et al. Oropharyngeal deglutition in stable COPD. Chest 2002; 121: 361-369.

5 Verin E, Clave P, Bonsignore MR, et al. Oropharyngeal dysphagia: when swallowing disorders meet respiratory diseases. Eur Respir J 2017; 49: 1602530.

6 Martin-Harris B, Brodsky MB, Michel Y, et al. Breathing and swallowing dynamics across the adult lifespan. Arch Otolaryngol Head Neck Surg 2005; 131: 762-770.

7 Cvejic L, Guiney N, Nicholson T, et al. Aspiration and severe exacerbations in COPD: a prospective study. ERJ Open Res 2021; 7: 00735-2020.

8 Terada K, Muro S, Ohara T, et al. Abnormal swallowing reflex and COPD exacerbations. Chest 2010; 137: 326-332.

9 Madhavan A, LaGorio LA, Crary MA, et al. Prevalence of and risk factors for dysphagia in the community dwelling elderly: a systematic review. J Nutr Health Aging 2016; 20: 806-815.

10 Butler JE, McKenzie DK, Crawford MR, et al. Role of airway receptors in the reflex responses of human inspiratory muscles to airway occlusion. J Physiol 1995; 487: 273-281.

11 Global Initiative for Chronic Obstructive Lung Disease (GOLD). Global Strategy for the Diagnosis, Management and Prevention of Chronic Obstructive Pulmonary Disease, 2020. Available from: www.goldcopd.org. Date last accessed: February 2020. Date last updated: 2020.

12 Epiu I, Gandevia SC, Boswell-Ruys CL, et al. Inspiratory muscle responses to sudden airway occlusion in chronic obstructive pulmonary disease. J Appl Physiol 2021; in press [https://doi.org/10.1152/japplphysiol. 00017.2021].

13 Miller MR, Hankinson J, Brusasco V, et al. Standardisation of spirometry. Eur Respir J 2005; 26: 319-338.

14 Quanjer PH, Stanojevic S, Cole TJ, et al. Multi-ethnic reference values for spirometry for the 3-95-yr age range: the global lung function 2012 equations. Eur Respir J 2012; 40: 1324-1343.

15 Belafsky PC, Mouadeb DA, Rees CJ, et al. Validity and reliability of the Eating Assessment Tool (EAT-10). Ann Otol Rhinol Laryngol 2008; 117: 919-924.

16 Cheney DM, Siddiqui MT, Litts JK, et al. The Ability of the 10-Item Eating Assessment Tool (EAT-10) to predict aspiration risk in persons with dysphagia. Ann Otol Rhinol Laryngol 2015; 124: 351-354.

17 IOPI Medical. Normal Values. Qualitative Guidelines for interpreting tongue elevation strength (Pmax). https:// iopimedical.com/normal-values/ Date last accessed: February 2020. Date last updated: 2020.

18 Hughes TA, Wiles CM. Clinical measurement of swallowing in health and in neurogenic dysphagia. Q J Med 1996; 89: 109-116.

19 Huckabee ML, McIntosh T, Fuller L, et al. The Test of Masticating and Swallowing Solids (TOMASS): reliability, validity and international normative data. Int J Lang Commun Disord 2018; 53: 144-156.

20 Murray NPS, McKenzie DK, Gorman RB, et al. Reproducibility of the short-latency reflex inhibition to loading of human inspiratory muscles. Respir Physio Neurobio 2008; 162: 216-222.

21 Nagami S, Oku Y, Yagi $N$, et al. Breathing-swallowing discoordination is associated with frequent exacerbations of COPD. BMJ Open Respir Res 2017; 4: e000202.

22 Gross RD, Atwood CW, Jr, Ross SB, et al. The coordination of breathing and swallowing in chronic obstructive pulmonary disease. Am J Respir Crit Care Med 2009; 179: 559-565.

23 Ghannouchi I, Speyer R, Doma K, et al. Swallowing function and chronic respiratory diseases: systematic review. Respir Med 2016; 117: 54-64.

24 Kobayashi S, Kubo H, Yanai M. Impairment of the swallowing reflex in exacerbations of COPD. Thorax 2007; 62: 1017.

25 O'Kane L, Groher M. Oropharyngeal dysphagia in patients with chronic obstructive pulmonary disease: a systematic review. Revista CEFAC 2009; 11: 449-506.

26 Zheng Z, Wu Z, Liu N, et al. Silent aspiration in patients with exacerbation of COPD. Eur Respir J 2016; 48: 570-573.

27 Singh B. Impaired swallow in COPD. Respirology 2011; 16: 185-186.

28 Cvejic L, Harding R, Churchward T, et al. Laryngeal penetration and aspiration in individuals with stable COPD. Respirology 2011; 16: 269-275.

29 Soler-Cataluna J, Martínez-García MÁ, Sánchez PR, et al. Severe acute exacerbations and mortality in patients with chronic obstructive pulmonary disease. Thorax 2005; 60: 925-931.

30 Seemungal TA, Donaldson GC, Paul EA, et al. Effect of exacerbation on quality of life in patients with chronic obstructive pulmonary disease. Am J Respir Crit Care Med 1998; 157: 1418-1422.

31 Nishino $\mathrm{T}$, Hasegawa R, Ide T, et al. Hypercapnia enhances the development of coughing during continuous infusion of water into the pharynx. Am J Respir Crit Care Med 1998; 157: 815-821.

32 Bautista TG, Sun Q-J, Pilowsky PM. The generation of pharyngeal phase of swallow and its coordination with breathing: interaction between the swallow and respiratory central pattern generators. Prog Brain Res 2014; 212: 253-275.

33 Ouahchy $\mathrm{Y}$, Messager V, Bon-Mardion N. Laryngeal implications in swallowing-ventilation coordination. Eur Respir J 2011; 38: Suppl. 55, A4404. 
34 Butler JE, McKenzie DK, Glanville AR, et al. Pulmonary afferents are not necessary for the reflex inhibition of human inspiratory muscles produced by airway occlusion. J Neurophysiol 1997; 78: 170-176.

35 Clayton NA, Carnaby GD, Peters MJ, et al. Impaired laryngopharyngeal sensitivity in patients with COPD: the association with swallow function. Int J Speech Lang Pathol 2014; 16: 615-623.

36 Chaves RdD, Carvalho CRFd, Cukier A, et al. Symptoms of dysphagia in patients with COPD. J Bras Pneumol 2011; 37: 176-183.

37 Feng $\mathrm{X}$, Todd $\mathrm{T}$, Lintzenich $\mathrm{CR}$, et al. Aging-related geniohyoid muscle atrophy is related to aspiration status in healthy older adults. J Gerontol A Biol Sci Med Sci 2013; 68: 853-860.

38 Aminpour S, Leonard R, Fuller SC, et al. Pharyngeal wall differences between normal younger and older adults. Ear Nose Throat J 2011; 90: E1.

39 Molfenter SM, Amin MR, Branski RC, et al. Age-related changes in pharyngeal lumen size: a retrospective MR analysis. Dysphagia 2015; 30: 321-327.

40 Martin-Harris B, Garand KLF, McFarland D. Optimizing respiratory-swallowing coordination in patients with oropharyngeal head and neck cancer. Perspect ASHA Spec Interest Groups 2017; 2: 103-110. 